\title{
Thermophilic bacteria are potential sources of novel Rieske non-heme iron oxygenases
}

\author{
Joydeep Chakraborty, Chiho Suzuki-Minakuchi, Kazunori Okada and Hideaki Nojiri* (i)
}

\begin{abstract}
Rieske non-heme iron oxygenases, which have a Rieske-type [2Fe-2S] cluster and a non-heme catalytic iron center, are an important family of oxidoreductases involved mainly in regio- and stereoselective transformation of a wide array of aromatic hydrocarbons. Though present in all domains of life, the most widely studied Rieske non-heme iron oxygenases are found in mesophilic bacteria. The present study explores the potential for isolating novel Rieske nonheme iron oxygenases from thermophilic sources. Browsing the entire bacterial genome database led to the identification of 45 homologs from thermophilic bacteria distributed mainly among Chloroflexi, Deinococcus-Thermus and Firmicutes. Thermostability, measured according to the aliphatic index, showed higher values for certain homologs compared with their mesophilic relatives. Prediction of substrate preferences indicated that a wide array of aromatic hydrocarbons could be transformed by most of the identified oxygenase homologs. Further identification of putative genes encoding components of a functional oxygenase system opens up the possibility of reconstituting functional thermophilic Rieske non-heme iron oxygenase systems with novel properties.
\end{abstract}

Keywords: Rieske non-heme iron oxygenase, Oxidoreductase, Thermophiles, Aromatic hydrocarbons,

Biotransformation

\section{Introduction}

Rieske non-heme iron oxygenases (ROs) constitute a large family of oxidoreductase enzymes involved primarily in the oxygenation of various aromatic compounds. Although Gibson et al. (1968) first detected the involvement of such an enzyme system in an alkylbenzenedegrading Pseudomonas sp., the family has since garnered a great deal of attention for two major reasons. First, ROs are key enzymes responsible for the initial attack on otherwise inert aromatic nuclei, thereby making them targets of a cascade of downstream enzymes, leading to their complete mineralization (Gibson and Subramanian 1984; Allen et al. 1995; Gibson and Parales 2000; Mallick et al. 2011). Secondly, regio- and stereoselective cis-dihydroxylation of aromatic compounds, catalyzed by ROs, generate impressive chiral intermediates in the synthesis

*Correspondence: anojiri@mail.ecc.u-tokyo.ac.jp Biotechnology Research Center, The University of Tokyo, 1-1-1 Yayoi, Bunkyo-ku, Tokyo, Japan of a wide array of agrochemically and pharmaceutically important compounds (Ensley et al. 1983; Wackett et al. 1988; Hudlicky et al. 1999; Bui et al. 2002; Newman et al. 2004; Boyd et al. 2005; Zezula and Hudlicky 2005).

Members of the RO family are usually either two- or three-component systems in which one or two soluble electron transport (ET) proteins (such as ferredoxin and reductase) transfer electrons from reduced nucleotides, such as $\mathrm{NAD}(\mathrm{P}) \mathrm{H}$, to the terminal oxygenase component (a large $\alpha$-subunit, often accompanied by a small $\beta$-subunit), which in turn catalyzes the di- or mono-oxygenation of the aromatic nucleus of the substrate (Mason and Cammack 1992; Ferraro et al. 2005). Numerous ROs have been identified and characterized from bacteria, thereby enriching the available information on their diversity in terms of both sequence and function (Habe and Omori 2003; Iwai et al. 2010, 2011; Chakraborty et al. 2012). Although found in all three domains of life, studies have shown that ROs occur more commonly in bacteria compared with archaea and eukaryotes (Chakraborty 
et al. 2012). Homologs of the large ( $\alpha$ ) subunit of RO terminal oxygenase $\left(\mathrm{RO}_{\mathrm{ox}}\right)$ have also been investigated in certain plant species, such as Arabidopsis thaliana, Zea mays, Pisum sativum, Oryza sativa, Physcomitrella patens, Amaranthus tricolor, Ocimum basilicum and Spinacia oleracea (Caliebe et al. 1997; Meng et al. 2001; Reinbothe et al. 2004; Berim et al. 2014), as well as in insects, nematodes and vertebrates (Rottiers et al. 2006; Yoshiyama et al. 2006; Yoshiyama-Yanagawa et al. 2011). ROs from these taxa, however, have entirely different functions from those of bacterial aromatic ring-hydroxylating ROs. They either act as proteintranslocons, facilitating transport across the chloroplastic envelope membranes during chlorophyll biosynthesis, or are involved in flavone and hormone metabolism in plants. They have also been suggested to be involved in regulation of cholesterol metabolism or trafficking during steroid synthesis in insects (Caliebe et al. 1997; Meng et al. 2001; Reinbothe et al. 2004; Rottiers et al. 2006; Yoshiyama et al. 2006; Yoshiyama-Yanagawa et al. 2011; Berim et al. 2014).

Interestingly, few bacterial RO homologs with novel functions, such as oxidative cyclization during biosynthesis of certain antibiotics, hydroxylation and desaturation of short-chain tertiary alcohols and alkane monooxygenation, have been reported in recent years (Sydor et al. 2011; Schäfer et al. 2012; Li et al. 2013). This suggests that ROs bear much more catalytic potential than previously realized. Almost all bacterial ROs characterized biochemically to date have been isolated from mesophilic bacteria, with the sole exception of polychlorinated biphenyl degrading ring-hydroxylating dioxygenase from Geobacillus sp. JF8 (Mukerjee-Dhar et al. 2005; Shintani et al. 2014). As such, very little is known about RO homologs present in bacteria that live in extreme environments. Extremophiles, and in particular their enzymes, have proved to be a potentially valuable resource in the development of novel biotechnological processes. The most well-studied extremophiles include thermophiles and hyperthermophiles, and enzymes isolated from such microorganisms are often extremely thermostable and resistant to proteolysis, chemical denaturants, detergents, and organic solvents (Vieille and Zeikus 2001). Apart from enzymatic stability at high temperatures, which is often desired in industrial processes, there are several advantages of thermophilic systems in bioremediation studies. Owing to the poor aqueous solubility of aromatic hydrocarbons, biodegradation studies often encounter problems related to bioavailability. These issues can be overcome at elevated temperatures, since bioavailability tends to increase with temperature owing to increases in solubility (Margesin and Schinner 2001; Feitkenhauer and Märk 2003; Perfumo et al. 2007). Thermophilic microorganisms may thus be attractive candidates for sources of novel thermostable ROs with potential utility in industrial biosynthesis and bioremediation at elevated temperatures.

In recent years, microbial genome sequencing projects have generated an enormous quantity of data for public databases. Since publication of the genome sequence of the first extremophile in 1996 (Bult et al. 1996), there has been a substantial increase in the number of extremophilic genome sequences. Metagenomics and single-cell genomics further add to this repertoire (Hedlund et al. 2014). The present study explored all available genome sequences of thermophilic bacteria for the presence of RO homologs and predicted their suitability as novel RO candidates for biotechnological applications.

\section{Materials and methods}

Screening thermophilic bacterial genomes for the presence of $\mathrm{RO}_{\mathrm{ox}}$ a-subunit homologs

Functionally characterized ROs have been categorized into five different similarity classes (A, B, C, D and D*) based on their phylogenetic distribution, substrate preferences and mode of attack on aromatic nuclei (Chakraborty et al. 2012). The National Center for Biotechnology Information (NCBI) 'genome' and 'taxonomy' databases were searched to characterize the distribution of thermophilic bacteria among different bacterial lineages and the availability of their genome sequences. Representative $\mathrm{RO}_{\text {ox }}$ $\alpha$-subunit sequences from each class were used as query probes (Table 1) to perform blastp (Altschul et al. 1990) searches against the translated set of genome sequences. Blast searches were also performed using each thermophilic RO as a query against the NCBI non-redundant database to characterize their distribution among thermophiles (and/or other extremophiles) and mesophiles.

\section{Phylogenetic clustering and prediction of substrate preferences}

The RHObase server (Chakraborty et al. 2014) was used to categorize each candidate thermophilic $\mathrm{RO}_{o x} \alpha$-subunit into a similarity class and to obtain the closest biochemically characterized homologs. The substrate prediction module of RHObase was further used to predict the substrate preference of the thermophilic homologs and the possible sites of oxygenation. ClustalX v1.81 (Thompson et al. 1997) was used to obtain multiple sequence alignments and to eliminate redundancy among sequences. The default settings were retained for all parameters, with the exception of the matrix (BLOSUM series) used for both pairwise and multiple alignments. Phylogenetic trees were constructed based on distance data using the neighborjoining method (Saitou and Nei 1987) implemented in ClustalX. The trees were visualized and manipulated using the program TreeExplorer v2.12 (Tamura et al. 2007). 
Table 1 List of queries used for blast analysis against genomes of thermophilic bacteria

\begin{tabular}{|c|c|c|c|c|}
\hline Target gene & Query & Type & Organism & GenBank accession no. \\
\hline \multirow[t]{5}{*}{ RO a-subunit } & Naphthalene dioxygenase (NahAc) & $A-\| l \mid a \beta$ & Pseudomonas putida NCIB 9816-4 & AAO64274 \\
\hline & Benzoate dioxygenase (BenA) & $B-\| a \beta$ & Rhodococcus jostii RHA1 & BAB70698 \\
\hline & Salicylate 5-hydroxylase (NagG) & $C-111 a \beta$ & Ralstonia sp. U2 & AAD12607 \\
\hline & Carbazole dioxygenase (CarAall) & D-Vlla & Sphingomonas sp. KA1 & YP_717942 \\
\hline & 3-Ketosteroid 9a-hydroxylase (KshA) & D-la & Mycobacterium tuberculosis H37rv & NP_218043 \\
\hline \multirow[t]{4}{*}{ Ferredoxin } & AntAb associated with anthranilate dioxygenase & Rieske type [2Fe-2S] & Sphingomonas sp. KA1 & YP_717959 \\
\hline & CarAcl associated with carbazole dioxygenase & Plant type [2Fe-2S] & Sphingomonas sp. KA1 & YP_717977 \\
\hline & PhtA3 associated with phthalate dioxygenase & [3Fe-4S] type & Terrabacter sp. DBF63 & BAC54160 \\
\hline & $\begin{array}{l}\text { Rub1 associated with naphthalene 1,2-dioxyge- } \\
\text { nase }\end{array}$ & Rubredoxin & Rhodococcus sp. P200 & AAR05110 \\
\hline \multirow[t]{5}{*}{ Reductase } & $\begin{array}{l}\text { KshB associated with 3-Ketosteroid } \\
\text { 9a-hydroxylase }\end{array}$ & FNRc type & Rhodococcus erythropolis PR4 & BAH32483 \\
\hline & CarAd associated with carbazole dioxygenase & FNRn type & Pseudomonas sp. XLDN4-9 & AAY56344 \\
\hline & Fdrl associated with carbazole dioxygenase & GR type & Sphingomonas sp. KA1 & YP_718026 \\
\hline & $\begin{array}{l}\text { HpaC associated with 4-hydroxyphenylacetate } \\
\text { 3-monooxygenase }\end{array}$ & Flavin reductase & Thermus thermophilus HB8 & 2ECU_A \\
\hline & Rubredoxin-NAD(+) reductase & Rubredoxin reductase & Pseudomonas aeruginosa PAO1 & Q9HTK9 \\
\hline
\end{tabular}

FNRc ferredoxin-NAD reductase fused with a plant-type [2Fe-2S] domain at the C-terminus, FNRn ferredoxin-NAD reductase fused with a plant-type [2Fe-2S] domain at the N-terminus, GR glutathione reductase

\section{Verification of the integrity of conserved motifs and domain architecture}

The $\mathrm{RO}_{\mathrm{ox}} \alpha$-subunit homologs obtained from the genomes of thermophiles were subjected to ScanProsite (De Castro et al. 2006) and NCBI conserved domain database searches (Marchler-Bauer et al. 2002) to verify the presence of conserved sequence motifs. The relevant motifs were $\mathrm{C}-\mathrm{X}-\mathrm{H}-\mathrm{X}_{\mathrm{n}}-\mathrm{C}-\mathrm{X}_{2}-\mathrm{H}$, corresponding to the $\mathrm{N}$-terminal Rieske $[2 \mathrm{Fe}-2 \mathrm{~S}]$ center, and $\mathrm{D}-\mathrm{X}_{2}-\mathrm{H}-\mathrm{X}_{3,4}-\mathrm{H}-$ $\mathrm{X}_{\mathrm{n}}-\mathrm{D}$, corresponding to the $\mathrm{C}$-terminal conserved 2-His1-carboxylate motif preceded by a conserved aspartate (involved in electron transport), as these are the functional prerequisites of ROs (Jiang et al. 1996; Parales 2003). The motifs were compared with those of phylogenetically close mesophilic ROs. For each protein, the aliphatic index (relative volume occupied by aliphatic side chains) (Ikai 1980) was calculated using ProtParam (Gasteiger et al. 2005).

\section{Identification of putative ET components}

Genomes exhibiting the presence of $\mathrm{RO}_{\mathrm{ox}} \alpha$-subunits were searched (using blastp) for genes putatively encoding ET components (both ferredoxin and reductase) using a set of queries (Table 1), followed by manual inspection of each genomic loci when necessary. The queries included the oxidoreductase sequences (e.g., ferredoxin-NAD reductases and glutathione reductasetype reductases and ferredoxins) commonly associated with ROs, as well as other possible oxidoreductases (e.g., flavin reductase and rubredoxin reductase).

\section{Results \\ Distribution of RO homologs among thermophiles}

Browsing the bacterial taxonomy database revealed the existence of several thermophilic genera belonging to different classes/orders. These taxa were concentrated mainly among the phyla Thermotogae, DeinococcusThermus, Chloroflexi, Aquificae, Firmicutes, and to some extent, Bacteroidetes/Chlorobi, Actinobacteria and Proteobacteria. Blast searches against all thermophile genomes initially led to the identification of 95 putative $\mathrm{RO}_{\text {ox }} \alpha$-subunit homologs distributed among 20 different genera (data not shown). Among 45 non-redundant sequences (Table 2), the one obtained from Alicyclobacillus acidoterrestris ATCC 49025 (GenBank: EPZ42375) was found to be truncated at the $\mathrm{N}$-terminal end and was therefore excluded from further analysis. Analysis of the distribution of the remaining candidate ROs among both thermophiles and mesophiles revealed that they were present mainly among thermophilic strains belonging to the phyla Chloroflexi, Deinococcus-Thermus, Firmicutes and Thermotogae (Fig. 1). However, distant homologs were abundant among mesophilic strains belonging to the phyla Actinobacteria, Firmicutes and Proteobacteria.

\section{Functional clustering of thermophilic $\mathrm{RO}_{\mathrm{ox}}$ a-subunit homologs}

The candidate $\alpha$-subunit sequences from thermophiles were subjected to phylogenetic studies to assess their relatedness with functionally characterized ROs from other bacteria, as well as from eukaryotes. The 
Table 2 Putative RO terminal oxygenase a-subunit homologues obtained from blastp search against thermophilic genomes

\begin{tabular}{|c|c|c|}
\hline Organism & Annotation & NCBI accn. no. \\
\hline \multicolumn{3}{|l|}{ Phylum: Chloroflexi } \\
\hline Anaerolinea thermophila UNI-1 & Putative oxidoreductase & BAJ63376 \\
\hline Caldilinea aerophila DSM 14535 & Putative oxidoreductase & BAL99910 \\
\hline Roseiflexus castenholzii DSM 13941 & Rieske (2Fe-2S) domain protein & ABU59830 \\
\hline Roseiflexus sp. RS-1 & (2Fe-2S)-binding protein & WP_011955741 \\
\hline Sphaerobacter thermophilus DSM 20745 & Rieske (2Fe-2S) domain protein & ACZ37596 \\
\hline Thermomicrobium roseum DSM 5159 & Rieske $2 \mathrm{Fe}-2 \mathrm{~S}$ domain protein & ACM04599 \\
\hline Thermomicrobium roseum DSM 5159 & 3-Phenylpropionate (digoxigenin) dioxygenase & ACM06903 \\
\hline \multicolumn{3}{|l|}{ Phylum: Deinococcus-Thermus } \\
\hline Meiothermus cerbereus DSM 11376 & Choline monooxygenase & WP_027876468 \\
\hline Meiothermus chliarophilus DSM 9957 & Choline monooxygenase & WP_027893118 \\
\hline Meiothermus ruber DSM 1279 & Aromatic-ring-hydroxylating dioxygenase, a-subunit & ADD29434 \\
\hline Meiothermus ruber DSM 1279 & Choline monooxygenase & AGK03951 \\
\hline Meiothermus rufus DSM 22234 & Hypothetical protein & WP_027881162 \\
\hline Meiothermus rufus DSM 22234 & Choline monooxygenase & WP_027882714 \\
\hline Meiothermus silvanus DSM 9946 & Rieske (2Fe-2S) iron-sulfur domain protein & ADH64647 \\
\hline Meiothermus timidus DSM 17022 & Choline monooxygenase & WP_018466224 \\
\hline Meiothermus timidus DSM 17022 & Ribosomal subunit interface protein & WP_026234685 \\
\hline Thermus igniterrae ATCC 700962 & Ribosomal subunit interface protein & WP_018111968 \\
\hline Thermus oshimai JL-2 & Ring-hydroxylating dioxygenase, large terminal subunit & AFV77211 \\
\hline Thermus scotoductus SA-01 & Biphenyl dioxygenase, subunit alpha & ADW21768 \\
\hline Thermus sp.CCB_US3_UF1 & Aromatic-ring-hydroxylating dioxygenase, a-subunit & AEV16357 \\
\hline Thermus thermophilus ATCC 33923 & Ribosomal subunit interface protein & WP_024119937 \\
\hline Thermus thermophilus JL-18 & Rieske (2Fe-2S) domain-containing protein & AFH40260 \\
\hline \multicolumn{3}{|l|}{ Phylum: Firmicutes } \\
\hline Alicyclobacillus acidocaldarius subsp. acidocaldarius DSM 446 & Rieske (2Fe-2S) iron-sulphur domain protein & ACV59062 \\
\hline Alicyclobacillus acidocaldarius subsp. acidocaldarius Tc-4-1 & $2 \mathrm{Fe}-2 \mathrm{~S}$ ferredoxin & AEJ44076 \\
\hline Alicyclobacillus acidoterrestris ATCC 49025 & Hypothetical protein & EPZ45189 \\
\hline Alicyclobacillus acidoterrestris ATCC 49025 & Hypothetical protein ${ }^{a}$ & EPZ42375 \\
\hline Alicyclobacillus hesperidum URH17-3-68 & 3-Phenylpropionate dioxygenase & WP_040289757 \\
\hline Alicyclobacillus pomorum DSM 14955 & Hypothetical protein & WP_035467417 \\
\hline Bacillus thermotolerans SGZ-8 & Phthalate 4,5-dioxygenase oxygenase subunit & KKB35183 \\
\hline Brevibacillus thermoruber PM1 & 3-Phenylpropionate dioxygenase & WP_035295329 \\
\hline Cohnella thermotolerans DSM 17683 & Rieske $(2 \mathrm{Fe}-2 \mathrm{~S})$ protein & WP_027092788 \\
\hline Coprothermobacter platensis DSM 11748 & Hypothetical protein & WP_018963776 \\
\hline Geobacillus sp. JF8 & Large subunit of biphenyl dioxygenase & AGT33881 \\
\hline Geobacillus thermoglucosidasius NBRC 107763 & Putative naphthalene 1,2-dioxygenase large subunit & GAJ45328 \\
\hline Sulfobacillus thermosulfidooxidans ST & Hypothetical protein & WP_051350961 \\
\hline Thermoactinomycetaceae bacterium GD1 & Rieske (2Fe-2S) protein & WP_044639983 \\
\hline Thermoanaerobacterium xylanolyticum LX-11 & Rieske (2Fe-2S) iron-sulfur domain protein & AEF16296 \\
\hline \multicolumn{3}{|l|}{ Phylum: Thermotogae } \\
\hline Fervidobacterium pennivorans DSM 9078 & Ring-hydroxylating dioxygenase, large terminal subunit & AFG35170 \\
\hline Thermosipho africanus TCF52B & Oxidase-related protein & ACJ75179 \\
\hline Thermotoga maritima MSB8 & Oxidase-related protein & AAD36358 \\
\hline Thermotoga maritima MSB8 & Rieske $(2 \mathrm{Fe}-2 \mathrm{~S})$ domain protein & AGL50271 \\
\hline Thermotoga naphthophila RKU-10 & Rieske (2Fe-2S) iron-sulphur domain protein & ADA67544 \\
\hline Thermotoga sp. Mc24 & Rieske (2Fe-2S) iron-sulfur domain-containing protein & KHC91410 \\
\hline Thermotoga sp. RQ2 & Rieske (2Fe-2S) domain protein & ACB09872 \\
\hline Thermotoga sp. Xyl54 & Rieske (2Fe-2S) iron-sulfur domain-containing protein & KHC95729 \\
\hline
\end{tabular}

a Protein truncated at the $\mathrm{N}$-terminal end 


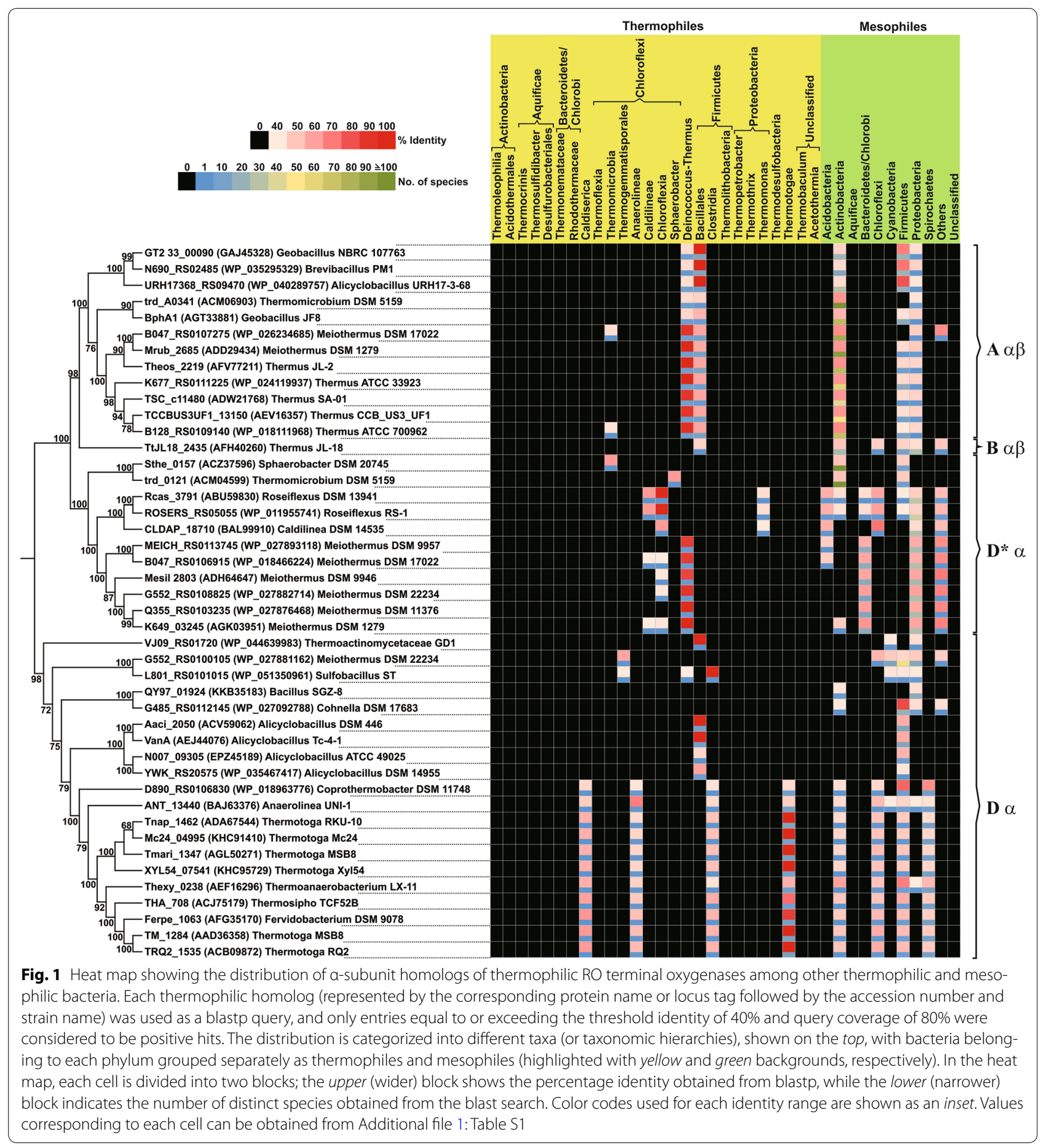

phylogenetic tree (Fig. 2) showed that thermophilic homologs were unevenly distributed among all classes of ROs, being clustered in a few specific regions of the tree, again suggesting a radical diversification followed by independent evolution of these genes in thermophiles. As can be seen in Fig. 2, 12 of the sequences clustered with Class A (especially Type A-V $\alpha \beta$ and A-VI $\alpha \beta$ ) ROs, 1 with Class B ROs, 20 with Class D ROs, and the remaining 11 with Class $D^{*}$ ROs. In all sequences, the conserved $\mathrm{N}$-terminal Rieske [2Fe-2S] motif and C-terminal 2-His1-carboxylate motif (preceded by a conserved aspartate involved in electron transport), necessary for proper 


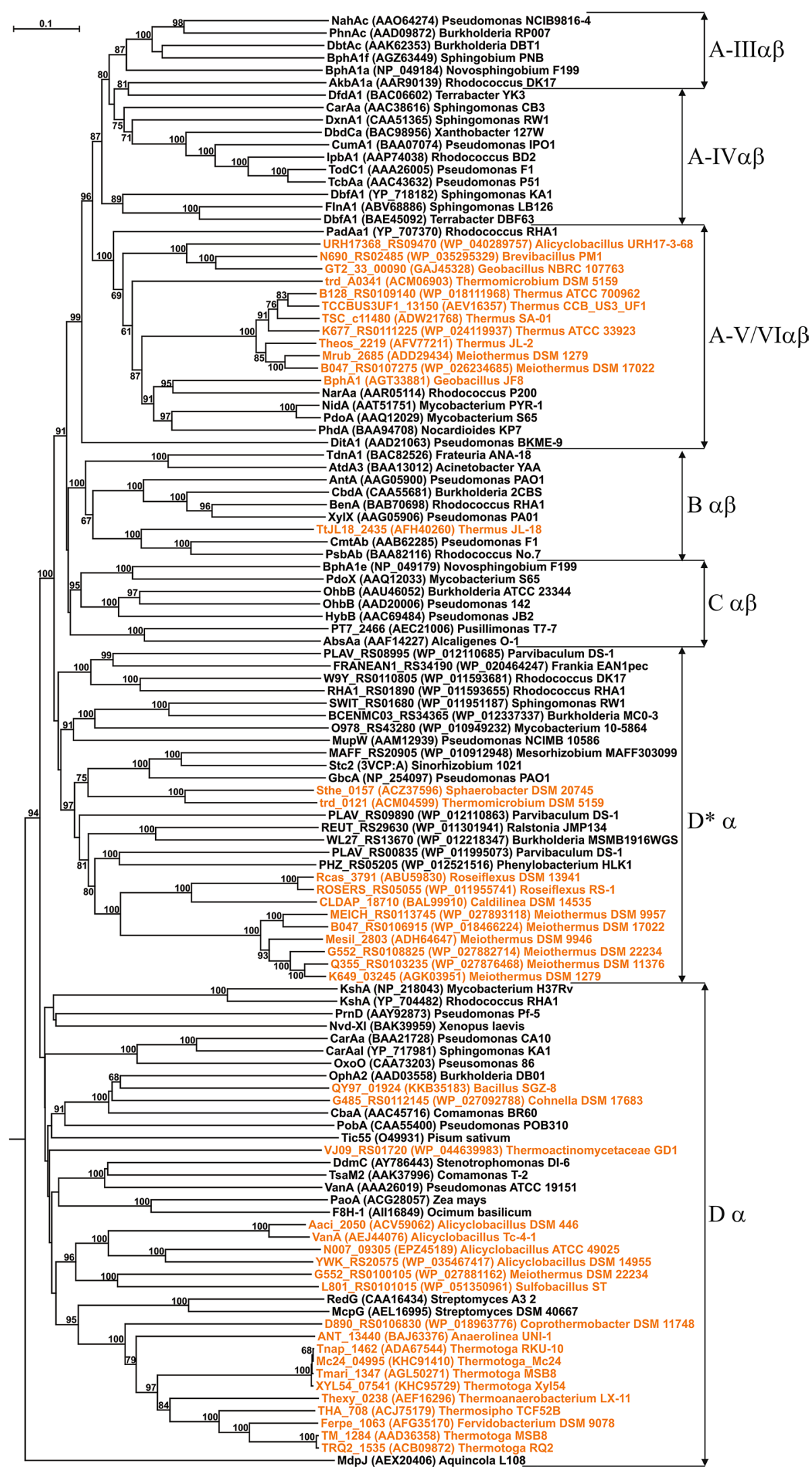


(See figure on previous page.)

Fig. 2 Neighbor-joining tree representing the phylogenetic relationship of putative a-subunits of oxygenase components of ROs obtained from thermophilic bacteria (orange font) with homologous sequences from other bacteria and eukaryotes. Each entry is represented by the corresponding protein name or locus tag, followed by the accession number (within parentheses) and the strain name. Values at each node indicate the level of bootstrap support based on 100 resampled datasets, while bootstrap values below $60 \%$ are not shown. The bar represents 0.1 substitutions per amino acid. The sequences have been clustered according to similarity class as defined in Chakraborty et al. (2014)

functioning of an RO (Jiang et al. 1996; Parales 2003), is consistent with phylogenetically related, previously characterized ROs (Fig. 3).

The aliphatic index is regarded as a positive factor for the increased thermostability of globular proteins (Ikai 1980). Therefore, we calculated this index for each protein as a measure of thermostability. Not all proteins showed significantly higher values compared with their mesophilic homologs (Fig. 3). However, the average value was found to be higher (80.88) in the thermophilic clusters as compared to those of the mesophiles (75.25), with some proteins (e.g., ROs obtained from Thermoactinomycetaceae bacterium GD1 [GenBank ID WP_044639983], Meiothermus rufus DSM 22234 [WP_027881162], Sphaerobacter thermophilus DSM 20745 [ACZ37596] and Thermomicrobium roseum DSM 5159 [ACM04599]) showing values as high as 90 (Fig. 3).

\section{Prediction of substrate preferences}

Table 3 lists the closest biochemically characterized homolog of each candidate $\mathrm{RO}_{\mathrm{ox}} \alpha$-subunit, as obtained from RHObase (Chakraborty et al. 2014). Preferable substrate(s) for most candidate ROs could be predicted using the RHObase substrate prediction module (Fig. 4). $\mathrm{RO}_{\mathrm{ox}} \alpha$-subunit homologs belonging to Class A showed a preference for polycyclic aromatic hydrocarbons and heterocyclic polyaromatic hydrocarbons. For one of the Class A ROs, obtained from Alicyclobacillus acidocaldarius subsp. acidocaldarius Tc-4-1, ketosteroid was found to be its putative substrate. The predicted substrates for the Class B $\mathrm{RO}_{\text {ox }} \alpha$-subunit from Thermus thermophilus JL-18 were carboxylated aromatics, such as $p$-cumate, while members of Class D showed a preference for carboxylated aromatics such as phthalate, chlorobenzoate, vanillate and phenoxybenzoates, as well as for toluene4-sulfonate. However, owing to the lack of information regarding the function of Class D* ROs, the substrate preference of these ROs could not be predicted. Apart from MupW and GbcA, involved in the mupirocin (ElSayed et al. 2003) and glycine betaine (Wargo et al. 2008) biosynthetic pathways, respectively, all other sequences belonging to this class have been derived from whole genome annotations and lack complete information regarding their biochemical function. This makes Class $D^{*}$ the 'dark matter' of Rieske oxygenases.

\section{Reconstitution of functional RO systems}

As discussed earlier, the oxygenase $\alpha$-subunit is often accompanied by a small $\beta$-subunit, and these subunits function in combination with one or two ET component(s). All observations discussed thus far concern the $\alpha$-subunits of $\mathrm{RO}_{\mathrm{ox}}$. However, to reconstitute a functional RO system, all of the above components must work together in a coordinated manner. Whenever present, the genes encoding both $\alpha$-and $\beta$-subunits are usually co-localized. Thus, the genome of each thermophile bearing the candidate $\mathrm{RO}_{\mathrm{ox}} \alpha$-subunit homologs was screened for the presence of genes putatively encoding the ET component(s). Several putative genes (listed in Table 4) were identified and, in most cases, were located at a distance from the terminal oxygenase genes. As expected, putative ferredoxin and reductase components, along with the $\beta$-subunit of $\mathrm{RO}_{\mathrm{ox}}$, could be identified in most organisms bearing Class A and B ROs. The genome of Thermus thermophilus ATCC 33923 had no adjacent $\beta$-subunit and did not yield any ferredoxin hits with the queries used. Alicyclobacillus hesperidum URH17-3-68 and Brevibacillus thermoruber PM1 were also found to lack ferredoxin. Among Class D ROs, D-IV $\alpha$ and D-VII $\alpha$ type ROs form three-component systems containing both ferredoxin and reductase (Chakraborty et al. 2012). Similarly, putative ferredoxin and reductase components could be identified in several thermophiles bearing Class D ROs. The only exceptions were Bacillus thermotolerans SGZ-8 and Thermoactinomycetaceae bacterium GD1,

\section{(See figure on next page.)}

Fig. 3 Comparison of conserved N-terminal Rieske [2Fe-2S] and C-terminal 2-His-1-carboxylate motifs among a-subunits of oxygenase components of putative thermophilic ROs obtained from thermophilic bacteria (orange font) and those obtained from mesophilic bacteria and eukaryotes. The horizontal bars represent the aliphatic index of each sequence. Blue and orange vertical dotted lines indicate the average aliphatic indices obtained for mesophilic (75.25) and thermophilic (80.88) RO homologs, respectively. All those homologs which showed an aliphatic index $\geq 80.88$ are indicated by an arrow, while clades representing only the thermophilic homologs are denoted by asterisks 


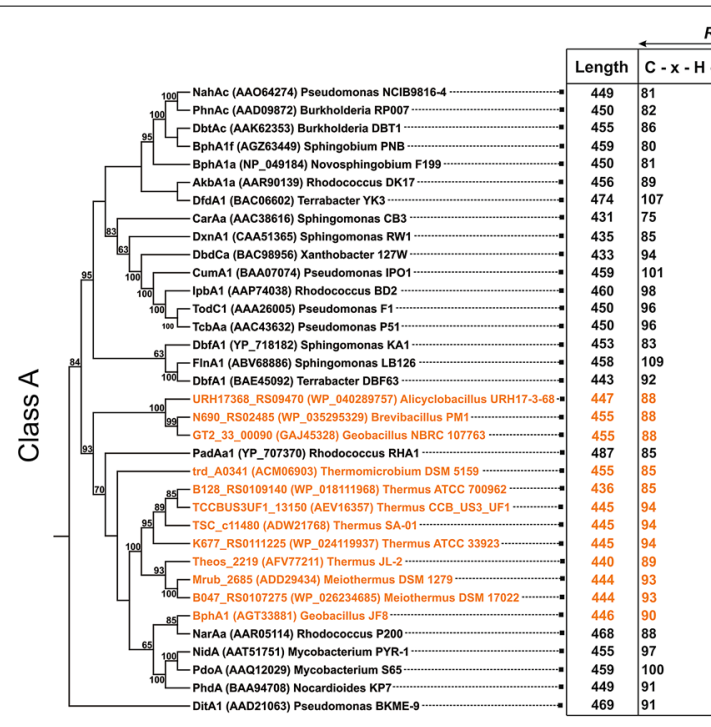

Rieske

2-His-1-carboxylate Aliphatic

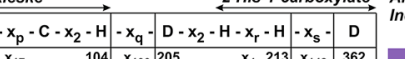

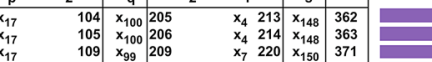

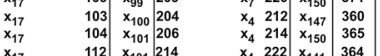

\begin{tabular}{lrr|r|r|}
$\mathrm{x}_{17}$ & 130 & $\mathrm{x}_{101}$ & 232 \\
$\mathrm{x}_{17}$ & 98 & $\mathrm{x}_{101}$ & 200 \\
$\mathrm{x}_{17}$ & 198 & $\mathrm{x}_{97}$ &
\end{tabular}

\begin{tabular}{lll|l|l|l}
$x_{17}$ & 108 & $x_{97}$ & 206 \\
$x_{17}$ & 117 & $x_{100}$ & 218 \\
$x_{17}$ & & 12 & $x_{17}$ & 231
\end{tabular}

\begin{tabular}{ll|l|l|l}
$\mathrm{x}_{17}$ & 124 & $\mathrm{x}_{106}$ & 231 \\
$\mathrm{x}_{17}$ & 121 & $\mathrm{x}_{99}$ & 221 \\
$\mathrm{x}_{17}$ & 119 & $\mathrm{x}_{17}$ & 219
\end{tabular}

\begin{tabular}{ll|l|l}
$x_{17}$ & 119 & $x_{99}$ & 219 \\
$x_{17}$ & 119 & $x_{99}$ & \\
$x_{17}$ & 100 & $x_{19}$ & 210
\end{tabular}

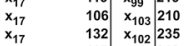

\begin{tabular}{lll|l|l}
$x_{17}$ & 115 & $x_{102}$ & 235 \\
$x_{102}$ & 218 \\
$x_{17}$ & 111 & $x_{17}$ &
\end{tabular}

\begin{tabular}{lll|l}
$x_{17}$ & 111 & $x_{101}$ & 213 \\
$x_{17}$ & 111 & $x_{101}$ & 213 \\
$x_{17}$ & 108 & $x_{102}$ & 211
\end{tabular}

\begin{tabular}{ll|l|l|l}
$x_{17}$ & 108 & $x_{102}$ & 21 \\
$x_{17}$ & 108 & $x_{100}$ & 20 \\
$x_{17}$ & 108 & $x_{10}$ & 210
\end{tabular}

\begin{tabular}{ll|l|l|l|l}
$x_{17}$ & 108 & $x_{101}$ & 210 \\
$x_{17}$ & 117 & $x_{101}$ & 219 \\
$x_{17}$ & 117 & $x_{101}$ & 219
\end{tabular}

\begin{tabular}{lll|l|l|l}
$x_{17}$ & 117 & $x_{101}$ & 219 \\
$x_{17}$ & 117 & $x_{101}$ & 219 \\
$x_{17}$ & 112 & $x_{10}$ & 214 \\
$x_{17}$ & 116 & $x_{10}$ & 19
\end{tabular}

\begin{tabular}{lll|l|l|l}
$x_{17}$ & & 112 & $x_{101}$ & 21 \\
$x_{17}$ & 116 & $x_{101}$ & 218 \\
$x_{17}$ & 116 & $x_{101}$ & 218 \\
$x_{17}$ & & & $x_{12}$ &
\end{tabular}

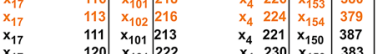

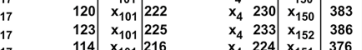

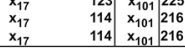

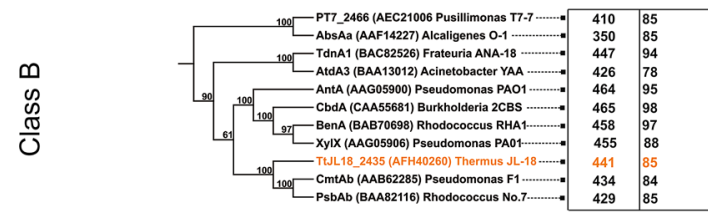

\begin{tabular}{|c|c|c|c|c|c|}
\hline$x_{17}$ & & \begin{tabular}{l|l|l}
$x_{96}$ & $E 205$
\end{tabular} & $\begin{array}{l}x_{3} \\
212\end{array}$ & $\mathrm{x}_{125}$ & \\
\hline$x_{17}$ & 108 & \begin{tabular}{l|l}
$\mathrm{x}_{97}$ & 206
\end{tabular} & & & \\
\hline$x_{16}$ & 116 & $\begin{array}{ll}x_{100} & 217\end{array}$ & $\mathrm{x}_{4} 225$ & $x_{147}$ & 373 \\
\hline (17) & $\begin{array}{l}101 \\
118\end{array}$ & \begin{tabular}{l|l}
$\mathbf{X}_{99}$ & 201
\end{tabular} & & \begin{tabular}{|l|}
$x_{146}$ \\
1
\end{tabular} & 356 \\
\hline$x_{1}$ & ${ }^{118}$ & $x_{100} 219$ & $\begin{array}{ll}x_{4} & 227\end{array}$ & \begin{tabular}{|l|}
$x_{153}$ \\
\end{tabular} & 381 \\
\hline$x_{17}$ & $\begin{array}{l}121 \\
120\end{array}$ & $\begin{array}{l}x_{102} \\
x_{12} 224 \\
223\end{array}$ & $\begin{array}{ll}x_{4} & 232 \\
x_{4} & 231\end{array}$ & $\begin{array}{l}x_{145} \\
x_{145}\end{array}$ & $\begin{array}{l}378 \\
378\end{array}$ \\
\hline $\begin{array}{l}x_{17} \\
\mathbf{x}_{17}\end{array}$ & 111 & \begin{tabular}{r|r|r|r|r|r|} 
& 250 \\
$\mathbf{x}_{102}$ & 214
\end{tabular} & $\begin{array}{r}x_{4} \\
x_{4} \\
2222\end{array}$ & $\begin{array}{l}x_{146} \\
\mathrm{x}_{146}\end{array}$ & 369 \\
\hline$x_{17}$ & 108 & \begin{tabular}{l|l|l}
$\mathrm{x}_{98}$ & 207
\end{tabular} & & $x_{161}$ & 377 \\
\hline & $\begin{array}{l}107 \\
108\end{array}$ & & & $x_{148}$ & \\
\hline & & & & & \\
\hline
\end{tabular}

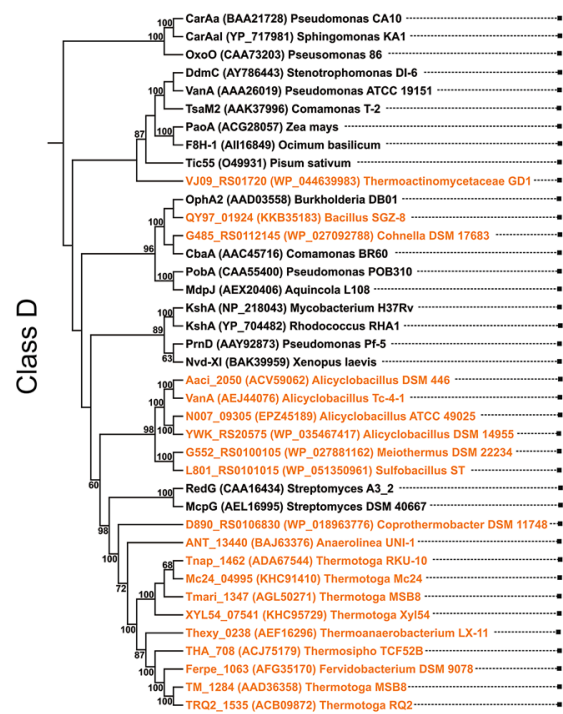

\begin{tabular}{|l|l|}
\hline 384 & 69 \\
378 & 69 \\
446 & 84 \\
339 & 48 \\
329 & 24 \\
346 & 47 \\
525 & 124 \\
523 & 123 \\
553 & 144 \\
354 & 55 \\
\hline 40 &
\end{tabular}

\begin{tabular}{l|l|l|l|l|}
93 & $\mathrm{x}_{36}$ & 180 \\
\hline
\end{tabular}

\begin{tabular}{ll|l|l|l|}
$\mathrm{x}_{4}$ & 222 & $\mathrm{x}_{141}$ & 364 \\
$\mathrm{x}_{4}$ & 240 & $\mathrm{x}_{142}$ & 383 \\
$\mathrm{x}_{4}$ & 208 & \\
$\mathrm{x}_{4}$ & 208 & $\mathrm{x}_{143}$ & 352 \\
$\mathrm{x}_{4}$ & 214 & $\mathrm{x}_{143}$ & 369
\end{tabular}

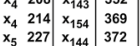

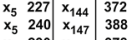

\begin{tabular}{ll|l|l|l|}
$x_{5}$ & 228 & $x_{147}$ & 376 \\
$x_{5}$ & 228 & $x_{147}$ & 376 \\
$x_{5}$ & 219 & $x_{169}$ & 389 \\
$x_{4}$
\end{tabular}

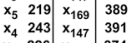

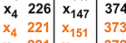

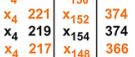

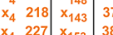

\begin{tabular}{ll|l|l|l}
$x_{4}$ & 227 & $x_{153}$ & 38 \\
$x_{4}$ & 227 & $x_{153}$ & 381 \\
$x_{4}$ & 227 & $x_{15}$ & 38 \\
$x_{4}$ & 22 & $x_{153}$ & 36
\end{tabular}

:

$\begin{array}{lll} & *\end{array}$ \begin{tabular}{lllll}
$\mathrm{x}_{4}$ & 224 & $\mathbf{x}_{151}$ & 376 \\
$\mathbf{x}_{4}$ & 224 & $x_{174}$ & 399 \\
\hline
\end{tabular}

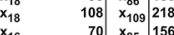

\begin{tabular}{lll|l|l}
$x_{16}$ & 70 & $x_{85}$ & 156 \\
$x_{16}$ & 46 & $x_{83}$ & 130 \\
$x_{16}$ & 69 & $x_{34}$ & 154 \\
$x_{16}$ & 14 & $x_{94}$ & 242
\end{tabular}

\begin{tabular}{r|r|r|}
69 & $x_{83}$ & 154 \\
147 & $x_{34}$ & 242 \\
149 & $x_{35}$ & 242
\end{tabular}

\begin{tabular}{ll|l|l|l}
146 & $x_{95}$ & 242 \\
166 & $x_{39}$ & 254 \\
7 & $x_{96}$ & 140
\end{tabular}

\begin{tabular}{r|r|r|}
166 & $x_{87}$ & 254 \\
77 & $x_{96}$ & 176
\end{tabular}

\begin{tabular}{l|l|l|l}
77 & $x_{98}$ & 176 \\
92 & $x_{85}$ & 178 \\
91 & $x_{5}$ & 178
\end{tabular}

\begin{tabular}{l|l|l}
91 & $x_{86}$ & 178 \\
91 & $x_{85}$ & 177 \\
\hline & $x_{85}$
\end{tabular}

\begin{tabular}{r|r|r}
91 & $x_{85}$ & 177 \\
107 & $x_{99}$ & 207 \\
9 & $x_{6}$ & 130
\end{tabular}

\begin{tabular}{r|r|r}
43 & $x_{96}$ & 130 \\
89 & $x_{36}$ & 178
\end{tabular}

\begin{tabular}{l|l|l}
89 & $x_{88}$ & 178 \\
91 & $x_{88}$ & 179 \\
91 & $x_{91}$ & 183
\end{tabular}

\begin{tabular}{r|r|r|}
91 & $x_{91}$ & 183 \\
181 & $x_{86}$ & 268 \\
\hline & $x_{3}$ &
\end{tabular}

\begin{tabular}{l|l|l}
82 & $x_{86}$ & 167 \\
93 & $x_{34}$ & 178 \\
68 & $x_{92}$ & 161
\end{tabular}

\begin{tabular}{l|l|l|l|}
68 & $x_{84}$ & 178 \\
38 & $x_{92}$ & 161 \\
38 & $x_{88}$ & 127 \\
7 & $x_{8}$ & 155
\end{tabular}

\begin{tabular}{l|l|l|l}
71 & $x_{83}$ & 155 \\
73 & $x_{79}$ & 153 \\
\hline & $x_{79}$ &
\end{tabular}

\begin{tabular}{ll|l|l}
69 & $x_{88}$ & $E 158$ \\
69 & $x_{86}$ & $E 156$ \\
6 & & 156
\end{tabular}

\begin{tabular}{l|l|l}
69 & $x_{87}$ & 157 \\
69 & $x_{84}$ & 154 \\
$x_{34}$ & $x_{34}$
\end{tabular}

\begin{tabular}{l|l|l}
69 & $x_{84}$ & 154 \\
67 & $x_{84}$ & 152 \\
64 & $x_{84}$ & 149
\end{tabular}

\begin{tabular}{l|l|l|l}
64 & $x_{84}$ & 149 \\
64 & $x_{84}$ & 149
\end{tabular}

\begin{tabular}{ll:lll}
$*$ \\
\hline 0 & 50 & & \\
\hline & & & \\
\hline
\end{tabular}

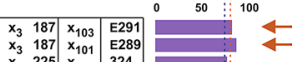

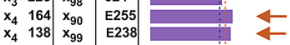

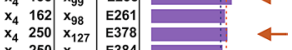

$\begin{array}{llll}\mathrm{x}_{4} & 262 & \mathbf{x}_{112} & 375 \\ x_{4} & & x_{4} & 250 \\ x_{4} & x_{13} & x_{96} & 273\end{array}$

\begin{tabular}{lll|l}
$x_{4}$ & 284 & $x_{11}$ & 375 \\
$x_{4}$ & 186 & $x_{86}$ & 273 \\
$x_{4}$ & $x_{36}$ &
\end{tabular}

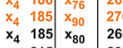

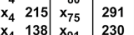

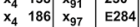

$\begin{array}{lllll}x_{4} & 187 & x_{97} & 284 \\ x_{4} & 191 & x_{96} & 285 \\ x_{93} & x_{93} & 285 \\ x_{4} & 276 & x_{92} & \end{array}$

\begin{tabular}{ll|l|l|l}
$\mathbf{x}_{4}$ & 276 & $\mathbf{x}_{82}$ & $\mathbf{E} 35$ \\
$\mathbf{x}_{4}$ & 175 & $\mathbf{x}_{64}$ & $\mathbf{E 2}$ \\
$\mathbf{x}_{4}$ & 186 & $x_{98}$ & 285 \\
$\mathbf{x}_{4}$ & & $x_{17}$ & 287
\end{tabular}

\begin{tabular}{lll|l|l|l}
$x_{4}$ & 186 & $x_{98}$ & 280 \\
$x_{4}$ & 169 & $x_{117}$ & 287 \\
$x_{4}$
\end{tabular}

× 254

\begin{tabular}{lll|l}
$x_{4}$ & 163 & $x_{111}$ & $E 262$ \\
$x_{4}$ & $*$ & $*$ & $*$
\end{tabular}

\begin{tabular}{lll|l}
$x_{4}$ & 161 & $x_{99}$ & 261 \\
$x_{4}$ & 166 & $x_{93}$ & 260
\end{tabular}

$\begin{array}{lllll}x_{4} & 666 & x_{93} & 260 \\ x_{4} & 164 & x_{91} & E 256\end{array}$

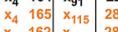

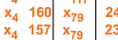

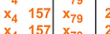

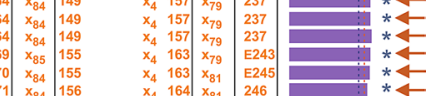

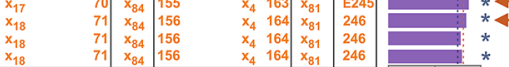

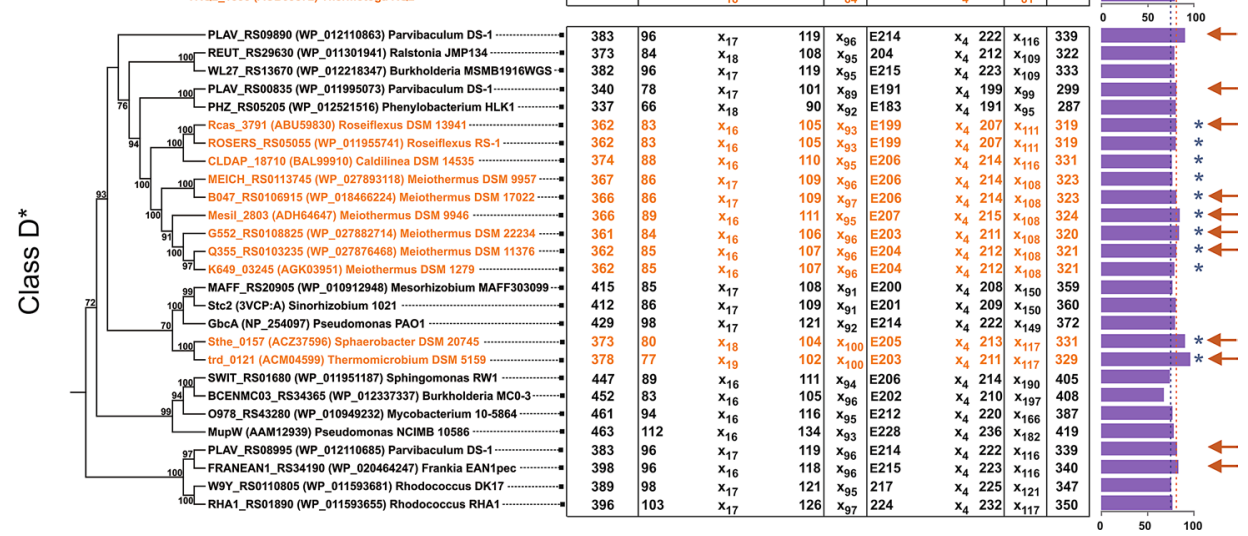


Table 3 The closest characterized homologue of each thermophilic RO terminal oxygenase a-subunit sequence obtained from RHObase

\begin{tabular}{l} 
Organism (NCBI accession no. of the putative \\
RO terminal oxygenase a-subunit) \\
\hline Class A \\
Thermomicrobium roseum DSM 5159 (ACM06903) \\
Meiothermus ruber DSM 1279 (ADD29434) \\
Meiothermus timidus DSM 17022 \\
(WP_026234685) \\
Thermus igniterrae ATCC 700962 (WP_018111968) \\
Thermus oshimai JL-2 (AFV77211) \\
Thermus scotoductus SA-01 (ADW21768) \\
Thermus sp. CCB_US3_UF1 (AEV16357) \\
Thermus thermophilus ATCC 33923 \\
(WP_024119937) \\
Alicyclobacillus hesperidum URH17-3-68 \\
(WP_040289757) \\
Brevibacillus thermoruber PM1 (WP_035295329) \\
Geobacillus sp. JF8 (AGT33881) \\
Geobacillus thermoglucosidasius NBRC 107763 \\
(GAJ45328) \\
Class B \\
Thermus thermophilus JL-18 (AFH40260) \\
Class D \\
Anaerolinea thermophila UNI-1 (BAJ63376) \\
The
\end{tabular}

Meiothermus rufus DSM 22234 (WP_027881162)

Closest match obtained from RHObase

Accession no.

Identity Query

(\%) coverage (\%)

Alicyclobacillus acidocaldarius subsp. acidocaldarius DSM 446 (ACV59062)

Alicyclobacillus acidocaldarius subsp. acidocaldarius TC-4-1 (AEJ44076)

Alicyclobacillus acidoterrestris ATCC 49025 (EPZ45189)

Alicyclobacillus pomorum DSM 14955 (WP_035467417)

Bacillus thermotolerans SGZ-8 (KKB35183)

Cohnella thermotolerans DSM 17683 (WP_027092788)

Coprothermobacter platensis DSM 11748 (WP_018963776)

Sulfobacillus thermosulfidooxidans ST (WP_051350961)

Thermoactinomycetaceae bacterium GD1 (WP_044639983)

Thermoanaerobacterium xylanolyticum LX-11 (AEF16296)

Fervidobacterium pennivorans DSM 9078 (AFG35170)

Thermosipho africanus TCF52B (ACJ75179)

Thermotoga maritima MSB8 (AAD36358)

Thermotoga maritima MSB8 (AGL50271)

Thermotoga naphthophila RKU-10 (ADA67544)

Biphenyl 2,3-dioxygenase (BphA1) from Geobacillus sp. JF8

Dibenzofuran dioxygenase (NarAa) from Rhodococcus opacus SAO101

Indene dioxygenase (NidA) from Rhodococcus sp. 124

Indene dioxygenase (NidA) from Rhodococcus sp. 124

Indene dioxygenase (NidA) from Rhodococcus sp. 124

Dibenzofuran dioxygenase (NarAa) from Rhodococcus opacus SAO101

Dibenzofuran dioxygenase (NarAa) from Rhodococcus opacus SAO101

Indene dioxygenase (NidA) from Rhodococcus sp. 124

Dibenzofuran dioxygenase (NarAa) from Rhodococcus opacus SA0101

Indene dioxygenase (NidA) from Rhodococcus sp. 124

Biphenyl 2,3-dioxygenase (BphA1) from Geobacillus sp. JF8

Indene dioxygenase (NidA) from Rhodococcus sp. 124

$\begin{array}{llr}\text { BAC79226 } & 46.10 & 91 \\ \text { BAD02377 } & 50.11 & 92 \\ & & \\ \text { AAD25395 } & 49.89 & 98 \\ & & 99 \\ \text { AAD25395 } & 51.48 & 98 \\ \text { AAD25395 } & 50.34 & 99 \\ \text { BAD02377 } & 51.14 & 97 \\ \text { BAD02377 } & 51.26 & 97 \\ \text { AAD25395 } & 51.03 & 93 \\ \text { BAD02377 } & 43.02 & 93 \\ \text { AAD25395 } & 44.59 & 92 \\ \text { BAC79226 } & 100.00 & 100 \\ \text { AAD25395 } & 45.81 & 93\end{array}$

p-Cumate dioxygenase (CmtAb) from Pseudomonas putida F1

$\begin{array}{lll}\text { AAB62285 } & 45.37 \quad 94\end{array}$

Dicamba O-demethylase (DdmC) from Stenotrophomonas maltophilia DI-6

Vanillate O-demethylase (VanA) from Pseudomonas sp. ATCC 1915

Toluene-4-sulfonate methyl monooxygenase (TsaM2) from Comamonas testosteroni T-2

3-Ketosteroid 9a-hydroxylase (KshA) from Rhodococcus opacus B-4

Toluene-4-sulfonate methyl monooxygenase (TsaM2) from Comamonas testosteroni $\mathrm{T}-2$

3-Chlorobenzoate-3,4/4,5-dioxygenase (CbaA) from Comamonas testosteroni BR60

Phthalate 4,5-dioxygenase (Pht3) from Pseudomonas putida

Phthalate 4,5-dioxygenase (OphA2) from Burkholderia cepacia $\mathrm{DBO} 1$

Dicamba O-demethylase (DdmC) from Stenotrophomonas maltophilia DI-6

Toluene-4-sulfonate methyl monooxygenase (TsaM2) from Comamonas testosteroni $\mathrm{T}-2$

Dicamba O-demethylase (DdmC) from Stenotrophomonas maltophilia DI-6

Vanillate O-demethylase (VanA) from Pseudomonas sp. HR199

AAV53699

$32.04 \quad 31$

AAA26019

$32.10 \quad 47$

AAK37996

32.45

AAK37996

$30.22 \quad 48$

$\begin{array}{lll}\text { AAC45716 } & 27.56 & 50\end{array}$

$\begin{array}{lll}\text { BAA02511 } & 37.86 & 94\end{array}$

$\begin{array}{lll}\text { AAD03558 } & 36.24 & 97\end{array}$

$\begin{array}{lll}\text { AAV53699 } & 32.93 & 51\end{array}$

$\begin{array}{lll}\text { AAK37996 } & 30.12 & 46\end{array}$

$\begin{array}{lll}\text { AAV53699 } & 34.78 & 54\end{array}$

$\begin{array}{lll}\text { CAA72287 } & 31.84\end{array}$

Dicamba O-demethylase (DdmC) from Stenotrophomonas maltophilia DI-6

Vanillate O-demethylase (VanA) from Pseudomonas sp. HR199

AAV53699

$34.13 \quad 47$

3-Chlorobenzoate-3,4/4,5-dioxygenase (CbaA) from Comamonas testosteroni BR60

Dicamba O-demethylase (DdmC) from Stenotrophomonas maltophilia DI-6

$\begin{array}{lll}\text { CAA72287 } & 33.92 & 46\end{array}$

AAC45716 $\quad 25.35 \quad 65$

AAV53699 $\quad 32.72 \quad 64$

$\begin{array}{llll}\text { Dicamba O-demethylase (DdmC) from Stenotrophomonas } & \text { AAV53699 } & 32.32 & 73\end{array}$ maltophilia DI-6

Dicamba O-demethylase (DdmC) from Stenotrophomonas maltophilia DI-6

$\begin{array}{lll}\text { AAV53699 } & 32.72 & 73\end{array}$ 
Table 3 continued

\begin{tabular}{|c|c|c|c|c|}
\hline $\begin{array}{l}\text { Organism (NCBI accession no. of the putative } \\
\text { RO terminal oxygenase } \alpha \text {-subunit) }\end{array}$ & Closest match obtained from RHObase & Accession no. & $\begin{array}{l}\text { Identity } \\
\text { (\%) }\end{array}$ & $\begin{array}{l}\text { Query } \\
\text { coverage (\%) }\end{array}$ \\
\hline Thermotoga sp. RQ2 (ACB09872) & $\begin{array}{l}\text { 3-Chlorobenzoate-3,4/4,5-dioxygenase (CbaA) from Comamonas } \\
\text { testosteroni BR60 }\end{array}$ & AAC45716 & 25.35 & 65 \\
\hline Thermotoga sp. Xyl54 (KHC95729) & $\begin{array}{l}\text { Dicamba O-demethylase (DdmC) from Stenotrophomonas } \\
\text { maltophilia DI-6 }\end{array}$ & AAV53699 & 32.72 & 73 \\
\hline \multicolumn{5}{|l|}{ Class D* } \\
\hline Caldilinea aerophila DSM 14535 (BAL99910) & 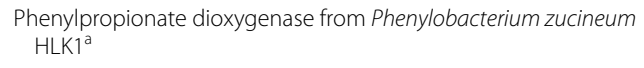 & WP_012521516 & 36.50 & 71 \\
\hline Roseiflexus castenholzii DSM 13941 (ABU59830) & $\begin{array}{l}\text { (2Fe-2S)-binding protein from Parvibaculum lavamentivorans } \\
\text { DS-1 }^{\mathrm{a}}\end{array}$ & WP_011995073 & 33.92 & 75 \\
\hline Roseiflexus sp. RS-1 (WP_011955741) & $\begin{array}{l}\text { (2Fe-2S)-binding protein from Parvibaculum lavamentivorans } \\
\text { DS-1T }\end{array}$ & WP_011995074 & 33.63 & 81 \\
\hline $\begin{array}{l}\text { Sphaerobacter thermophilus DSM } 20745 \\
\text { (ACZ37596) }\end{array}$ & Aromatic oxygenase (GbcA) from Pseudomonas aeruginosa PAO1 & NP_254097 & 31.36 & 88 \\
\hline Thermomicrobium roseum DSM 5159 (ACM04599) & Aromatic oxygenase (GbcA) from Pseudomonas aeruginosa PAO1 & NP_254097 & 29.47 & 91 \\
\hline $\begin{array}{l}\text { Meiothermus cerbereus DSM } 11376 \\
\text { (WP_027876468) }\end{array}$ & $\begin{array}{l}\text { (2Fe-2S)-binding protein from Parvibaculum lavamentivorans } \\
\text { DS-1 } 1 T^{a}\end{array}$ & WP_011995073 & 26.91 & 80 \\
\hline $\begin{array}{l}\text { Meiothermus chliarophilus DSM } 9957 \\
\text { (WP_027893118) }\end{array}$ & $\begin{array}{l}\text { (2Fe-2S)-binding protein from Parvibaculum lavamentivorans } \\
\text { DS-1T }\end{array}$ & WP_011995073 & 28.25 & 77 \\
\hline Meiothermus ruber DSM 1279 (AGK03951) & $\begin{array}{l}\text { Rieske }(2 \mathrm{Fe}-2 \mathrm{~S}) \text { domain-containing protein from Parvibaculum } \\
\text { lavamentivorans DS-1 }{ }^{\mathrm{a}}\end{array}$ & WP_011995073 & 40.68 & 76 \\
\hline Meiothermus rufus DSM 22234 (WP_027882714) & $\begin{array}{l}\text { Rieske }(2 \mathrm{Fe}-2 \mathrm{~S}) \text { domain-containing protein from Parvibaculum } \\
\text { lavamentivorans DS-1 } 1^{\mathrm{a}}\end{array}$ & WP_011995073 & 26.84 & 84 \\
\hline Meiothermus silvanus DSM 9946 (ADH64647) & $\begin{array}{l}\text { Rieske }(2 \mathrm{Fe}-2 \mathrm{~S}) \text { domain-containing protein from Parvibaculum } \\
\text { lavamentivorans DS-1 } 1^{\text {a }}\end{array}$ & WP_011995073 & 29.83 & 80 \\
\hline $\begin{array}{l}\text { Meiothermus timidus DSM } 17022 \\
\text { (WP_018466224) }\end{array}$ & $\begin{array}{l}\text { Rieske }(2 \mathrm{Fe}-2 \mathrm{~S}) \text { domain-containing protein from Parvibaculum } \\
\text { lavamentivorans DS-1 }{ }^{\text {a }}\end{array}$ & WP_011995073 & 27.81 & 78 \\
\hline
\end{tabular}

${ }^{a}$ Biochemically uncharacterized protein

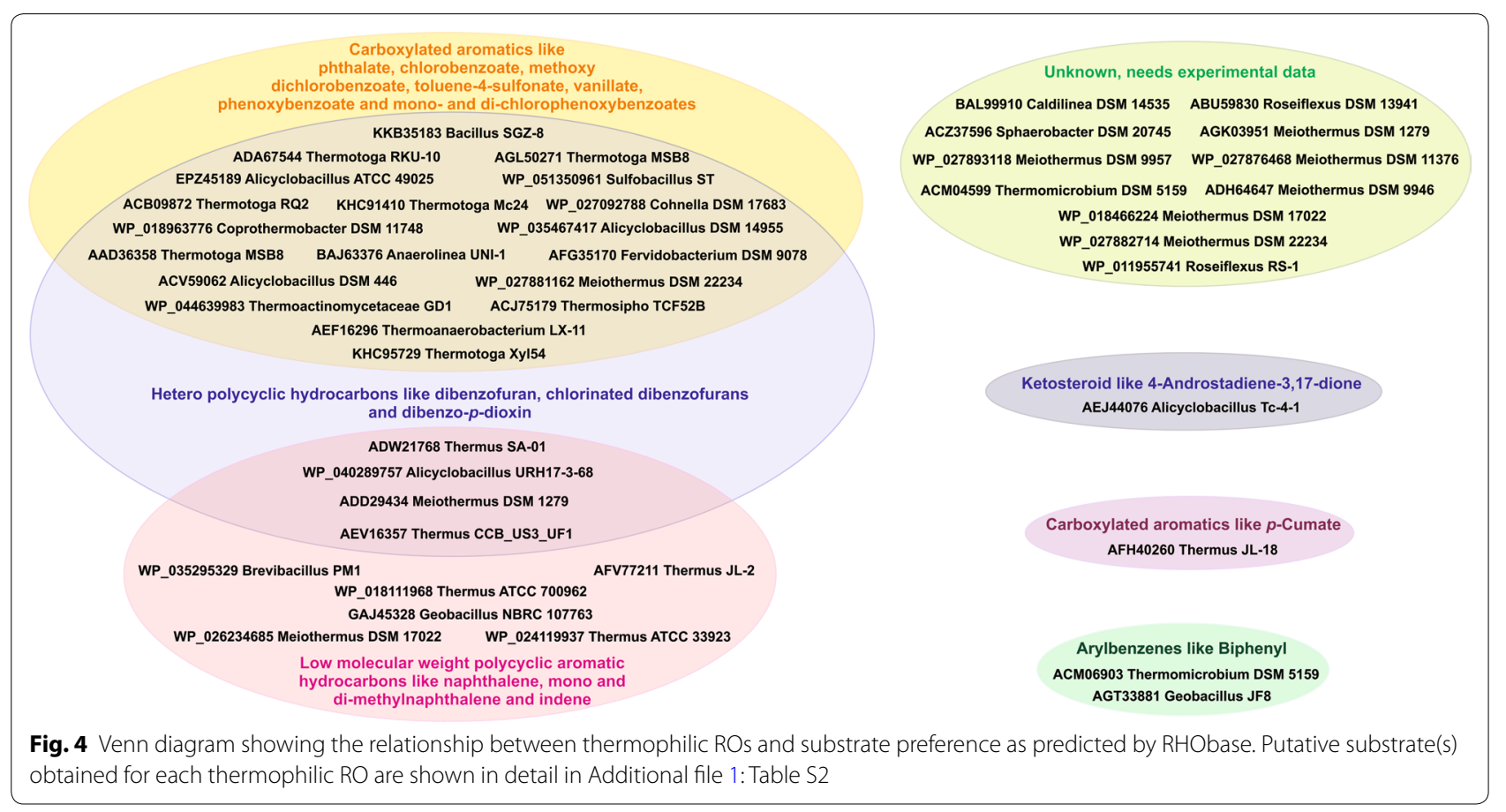


Table 4 Constituent components, obtained from each thermophile genome, that can be coupled with a-subunit of the terminal oxygenase component in order to reconstitute functional RO system

\begin{tabular}{|c|c|c|}
\hline $\begin{array}{l}\text { Organism (NCBI accession no. of the putative RO } \\
\text { terminal oxygenase a-subunit) }\end{array}$ & Protein annotation [accession no.] & $\begin{array}{l}\text { Putative func- } \\
\text { tion }\end{array}$ \\
\hline \multicolumn{3}{|l|}{ Class A } \\
\hline \multirow[t]{3}{*}{ Thermomicrobium roseum DSM 5159 (ACM06903) } & 3-Phenylpropionate dioxygenase subunit beta [ACM06517] & Beta subunit \\
\hline & $\begin{array}{l}\text { Rieske protein [ACM07109] } \\
\text { 4Fe-4S ferredoxin, iron-sulfur binding domain protein [ACM06911] }\end{array}$ & Ferredoxin \\
\hline & $\begin{array}{l}\text { Ferredoxin-NADP reductase; thioredoxin reductase [ACM06977] } \\
\text { Putidaredoxin reductase [ACM07176] } \\
\text { Thioredoxin reductase [ACM06488] }\end{array}$ & Reductase \\
\hline \multirow[t]{3}{*}{ Meiothermus ruber DSM 1279 (ADD29434) } & Aromatic-ring-hydroxylating dioxygenase beta subunit [ADD29433] & Beta subunit \\
\hline & Rieske (2Fe-2S) domain protein [ADD27546] & Ferredoxin \\
\hline & Dihydrolipoamide dehydrogenase [ADD29074] & Reductase \\
\hline \multirow[t]{3}{*}{ Meiothermus timidus DSM 17022 (WP_026234685) } & Aromatic-ring-hydroxylating dioxygenase subunit beta [WP_018466297] & Beta subunit \\
\hline & Diguanylate cyclase [WP_026234838] & Ferredoxin \\
\hline & $\begin{array}{l}\text { Dihydrolipoamide dehydrogenase [WP_026234742] } \\
\text { Dihydrolipoamide dehydrogenase [WP_026234716] } \\
\text { hypothetical protein [WP_018465799] }\end{array}$ & Reductase \\
\hline Thermus igniterrae ATCC 700962 (WP_018111968) & Aromatic-ring-hydroxylating dioxygenase subunit beta [WP_018111967] & Beta subunit \\
\hline \multirow[t]{3}{*}{ Thermus oshimai JL-2 (AFV77211) } & Small subunit of phenylpropionate dioxygenase [AFV77212] & Beta subunit \\
\hline & $\begin{array}{l}\text { Ferredoxin subunit of nitrite reductase and ring-hydroxylating dioxygenase } \\
\text { [AFV75193] }\end{array}$ & Ferredoxin \\
\hline & $\begin{array}{l}\text { NAD(FAD)-dependent dehydrogenase [AFV75199] } \\
\text { Dihydrolipoamide dehydrogenase [AFV77091] } \\
\text { Flavoprotein oxygenase [WP_016329217] }\end{array}$ & Reductase \\
\hline \multirow[t]{3}{*}{ Thermus scotoductus SA-01 (ADW21768) } & 3-Phenylpropionate dioxygenase, subunit beta [ADW21767] & Beta subunit \\
\hline & $\begin{array}{l}\text { Ferredoxin subunit of phenylpropionate dioxygenase [ADW21026] } \\
\text { 4Fe-4S Ferredoxin/formate dehydrogenase, nitrate-inducible, iron-sulfur } \\
\text { subunit [ADW21629] }\end{array}$ & Ferredoxin \\
\hline & $\begin{array}{l}\text { NADH oxidase/coenzyme A disulfide reductase [ADW21032] } \\
\text { Phenol hydroxylase component B [ADW21668] }\end{array}$ & Reductase \\
\hline \multirow[t]{3}{*}{ Thermus sp. CCB_US3_UF1 (AEV16357) } & Naphthalene dioxygenase small subunit [AEV16358] & Beta subunit \\
\hline & Initial dioxygenase ferredoxin subunit [AEV16981] & Ferredoxin \\
\hline & $\begin{array}{l}\text { 4-Hydroxybenzoate 3-monooxygenase [AEV16360] } \\
\text { FAD-dependent pyridine nucleotide-disulfide oxidoreductase [AEV16975] } \\
\text { 4-Hydroxyphenylacetate 3-monooxygenase reductase (Flav_red) [AEV16285] }\end{array}$ & Reductase \\
\hline $\begin{array}{l}\text { Thermus thermophilus ATCC } 33923 \\
\text { (WP_024119937) }\end{array}$ & NADH oxidase [WP_024119527] & Reductase \\
\hline \multirow{2}{*}{$\begin{array}{l}\text { Alicyclobacillus hesperidum URH17-3-68 } \\
\text { (WP_040289757) }\end{array}$} & Thioesterase superfamily protein [EJY55215] & Beta subunit \\
\hline & $\begin{array}{l}\text { Dihydropteridine reductase [WP_006448064] } \\
\text { Dihydrolipoamide dehydrogenase [WP_006446492] } \\
\text { NADH dehydrogenase [WP_006448257] } \\
\text { Thioredoxin reductase [WP_006447474]; }\end{array}$ & Reductase \\
\hline \multirow[t]{2}{*}{ Brevibacillus thermoruber PM1 (WP_035295329) } & Aromatic-ring-hydroxylating dioxygenase [WP_035295713] & Beta subunit \\
\hline & Dihydrolipoamide dehydrogenase [WP_035297436] & Reductase \\
\hline \multirow[t]{3}{*}{ Geobacillus sp. JF8 (AGT33881) } & Small subunit of biphenyl dioxygenase [AGT33882] & Beta subunit \\
\hline & Nitrite reductase NAD(P)H small subunit [AGT32189] & Ferredoxin \\
\hline & Thioredoxin reductase [AGT33412] & Reductase \\
\hline \multirow{3}{*}{$\begin{array}{l}\text { Geobacillus thermoglucosidasius NBRC } 107763 \\
\text { (GAJ45328) }\end{array}$} & Putative naphthalene 1,2-dioxygenase small subunit [GAJ45329] & Beta subunit \\
\hline & Hypothetical protein GT2_34_00240 [GAJ45383] & Ferredoxin \\
\hline & $\begin{array}{l}\text { Thioredoxin reductase [GAJ43870] } \\
\text { Coenzyme A disulfide reductase [GAJ42754] } \\
\text { Putative oxidoreductase [GAJ45381] }\end{array}$ & Reductase \\
\hline
\end{tabular}


Table 4 continued

Organism (NCBI accession no. of the putative RO Protein annotation [accession no.]

Putative functerminal oxygenase a-subunit)

tion

Class B

Thermus thermophilus JL-18 (AFH40260)

Class D

Anaerolinea thermophila UNI-1 (BAJ63376)

Meiothermus rufus DSM 22234 (WP_027881162)

Alicyclobacillus acidocaldarius subsp. acidocaldarius DSM 446 (ACV59062)

Alicyclobacillus acidocaldarius subsp. acidocaldarius TC-4-1 (AEJ44076)

Alicyclobacillus acidoterrestris ATCC 49025 (EPZ45189)

Alicyclobacillus pomorum DSM 14955 (WP_035467417)

Bacillus thermotolerans SGZ-8 (KKB35183)

Cohnella thermotolerans DSM 17683 (WP_027092788)

Coprothermobacter platensis DSM 11748 (WP_018963776)

Sulfobacillus thermosulfidooxidans ST (WP_051350961)

Thermoactinomycetaceae bacterium GD1 (WP_044639983)

Thermoanaerobacterium xylanolyticum LX-11 (AEF16296)

Fervidobacterium pennivorans DSM 9078 (AFG35170)

Thermosipho africanus TCF52B (ACJ75179)

Thermotoga maritima MSB8 (AAD36358)

Thermotoga maritima MSB8 (AGL50271)

Thermotoga naphthophila RKU-10 (ADA67544)
Small subunit of phenylpropionate dioxygenase [AFH40261]

Ferredoxin subunit of nitrite reductase and ring-hydroxylating dioxygenase [AFH39800]

NAD(FAD)-dependent dehydrogenase [AFH39794]

NAD(FAD)-dependent dehydrogenase [AFH40137]

Putative ferredoxin [BAJ63818]

Phytoene dehydrogenase [BAJ63379]

Dehydrogenase [WP_027881168]

Rieske (2Fe-2S) iron-sulphur domain protein [ACV58029]

Arsenate reductase (thioredoxin) [ACV59045]

Flavin reductase domain protein FMN-binding [ACV59470]

Flavin reductase domain protein FMN-binding [ACV58921]

Rieske (2Fe-2S) domain protein [AEJ42957]

Dihydrolipoamide dehydrogenase [AEJ42545]

Flavin reductase domain protein FMN-binding protein [AEJ43919]

Hypothetical protein N007 08015 [EPZ45711]

Hypothetical protein [EPZ45711]

Dihydropteridine reductase [WP_035467306]

Beta subunit

Ferredoxin

Reductase

Ferredoxin

Reductase

Reductase

Ferredoxin

Reductase

Ferredoxin

Reductase

Reductase

Reductase

Absent

Ferredoxin [WP 027093665]

Ferredoxin

2-Enoate reductase [WP_018963737]

Hypothetical protein [WP_018963777]

(2Fe-2S)-binding protein [WP_037913512]

Hypothetical protein, partial [WP_040767264]

Absent

Rubredoxin-type $\mathrm{Fe}(\mathrm{Cys})_{4}$ protein [AEF16485]

Ferredoxin

Reductase

CoA-disulfide reductase [AEF16270]

Thioredoxin reductase [AEF16306]

Rubredoxin [AFG34604]

2-Polyprenylphenol hydroxylase-like oxidoreductase [AFG34955]

Phytoene dehydrogenase-like oxidoreductase [AFG35173]

Thioredoxin-disulfide reductase [AFG35278]

FAD binding protein [AFG35124]

Thioredoxin reductase [AFG34984]

Dihydrolipoamide dehydrogenase [AFG34941]

Thioredoxin reductase [AFG35081]

Rubredoxin [ACJ76024]

Ferredoxin

Oxidoreductase/NADH: ubiquinone oxidoreductase, na translocating, f-subunit [ACJ75165]

Oxidoreductase/tRNA uridine 5-carboxymethylaminomethyl modification enzyme GidA [ACJ75137]

Ferredoxin/anaerobic glycerol 3-phosphate dehydrogenase [ACJ75136]

Rubredoxin [AGL49584]

Oxidoreductase [AAD35836]

Rubredoxin [AGL49584]

Oxidoreductase [AAD35836]

Rubredoxin-type $\mathrm{Fe}(\mathrm{Cys})_{4}$ protein [ADA66551]

FAD-dependent pyridine nucleotide-disulphide oxidoreductase [ADA67462]
Reductase

Ferredoxin

Reductase

Ferredoxin

Reductase

Ferredoxin

Reductase 
Table 4 continued

\begin{tabular}{|c|c|c|}
\hline $\begin{array}{l}\text { Organism (NCBI accession no. of the putative RO } \\
\text { terminal oxygenase } a \text {-subunit) }\end{array}$ & Protein annotation [accession no.] & $\begin{array}{l}\text { Putative func- } \\
\text { tion }\end{array}$ \\
\hline \multirow[t]{2}{*}{ Thermotoga sp. Mc24 (KHC91410) } & Rubredoxin-type $\mathrm{Fe}(\mathrm{Cys})_{4}$ protein [KHC90328] & Ferredoxin \\
\hline & Oxidoreductase [KHC90231] & Reductase \\
\hline \multirow[t]{2}{*}{ Thermotoga sp. RQ2 (ACB09872) } & Rubredoxin-type $\mathrm{Fe}(\mathrm{Cys})_{4}$ protein [ACB08629] & Ferredoxin \\
\hline & FAD-dependent pyridine nucleotide-disulphide oxidoreductase [ACB08534] & Reductase \\
\hline Thermotoga sp. Xyl54 (KHC95729) & Rubredoxin-type $\mathrm{Fe}(\mathrm{Cys})_{4}$ protein [KHC96420] & Ferredoxin \\
\hline \multicolumn{3}{|l|}{ Class $D^{*}$} \\
\hline \multirow[t]{2}{*}{ Caldilinea aerophila DSM 14535 (BAL99910) } & Hypothetical protein [BAL99023] & Ferredoxin \\
\hline & $\begin{array}{l}\text { Putative mercuric reductase [BAM01871] } \\
\text { Putative flavin reductase [BAL99068] }\end{array}$ & Reductase \\
\hline \multirow[t]{2}{*}{ Roseiflexus castenholzii DSM 13941 (ABU59830) } & Rieske (2Fe-2S) domain protein [ABU56444] & Ferredoxin \\
\hline & $\begin{array}{l}\text { Dihydrolipoamide dehydrogenase [ABU58189] } \\
\text { Flavin reductase domain protein FMN-binding [ABU60217] } \\
\text { Flavin reductase domain protein FMN-binding [ABU58489] }\end{array}$ & Reductase \\
\hline \multirow[t]{2}{*}{ Roseiflexus sp. RS-1 (WP_011955741) } & Rieske (2Fe-2S) domain protein [ABQ89132] & Ferredoxin \\
\hline & $\begin{array}{l}\text { FAD-dependent pyridine nucleotide-disulphide oxidoreductase [ABQ91503] } \\
\text { Dihydrolipoamide dehydrogenase [ABQ91367] } \\
\text { Flavin reductase domain protein, FMN-binding [ABQ92660] } \\
\text { Flavin reductase domain protein, FMN-binding [ABQ90950] }\end{array}$ & Reductase \\
\hline \multirow{2}{*}{$\begin{array}{l}\text { Sphaerobacter thermophilus DSM } 20745 \\
\text { (ACZ37596) }\end{array}$} & Rieske (2Fe-2S) iron-sulphur domain protein [ACZ40633] & Ferredoxin \\
\hline & $\begin{array}{l}\text { FAD-dependent pyridine nucleotide-disulphide oxidoreductase [ACZ40632] } \\
\text { Flavin reductase domain protein FMN-binding protein [ACZ39977] }\end{array}$ & Reductase \\
\hline \multirow[t]{2}{*}{ Thermomicrobium roseum DSM 5159 (ACM04599) } & Rieske protein [ACM07109] & Ferredoxin \\
\hline & $\begin{array}{l}\text { Xylene monooxygenase electron transfer subunit [ACM06651] } \\
\text { Ferredoxin-NADP reductase; thioredoxin reductase [ACM06977] } \\
\text { Putidaredoxin reductase [ACM07176] } \\
\text { Thioredoxin reductase [ACM06488] }\end{array}$ & Reductase \\
\hline \multirow{2}{*}{$\begin{array}{l}\text { Meiothermus cerbereus DSM } 11376 \\
\text { (WP_027876468) }\end{array}$} & Diguanylatecyclase [WP_027876040] & Ferredoxin \\
\hline & $\begin{array}{l}\text { Dihydrolipoyl dehydrogenase [WP_027876580] } \\
\text { MFS transporter [WP_027877386] }\end{array}$ & Reductase \\
\hline $\begin{array}{l}\text { Meiothermus chliarophilus DSM } 9957 \\
\text { (WP_027893118) }\end{array}$ & $\begin{array}{l}\text { Dihydrolipoyl dehydrogenase [WP_027893501] } \\
\text { MFS transporter [WP_036218580] }\end{array}$ & Reductase \\
\hline Meiothermus ruber DSM 1279 (AGK03951) & $\begin{array}{l}\text { Dihydrolipoamide dehydrogenase [ADD29074] } \\
\text { Flavin reductase domain-containing FMN-binding protein [AGK04562] }\end{array}$ & Reductase \\
\hline Meiothermus rufus DSM 22234 (WP_027882714) & Dehydrogenase [WP_027881168] & Reductase \\
\hline \multirow[t]{2}{*}{ Meiothermus silvanus DSM 9946 (ADH64647) } & Rieske (2Fe-2S) iron-sulfur domain protein [ADH62911] & Ferredoxin \\
\hline & $\begin{array}{l}\text { FAD-dependent pyridine nucleotide-disulfide oxidoreductase [ADH64702] } \\
\text { Flavin reductase domain protein FMN-binding protein [ADH64172] }\end{array}$ & Reductase \\
\hline Meiothermus timidus DSM 17022 (WP_018466224) & $\begin{array}{l}\text { Dihydrolipoamide dehydrogenase [WP_026234742] } \\
\text { Dihydrolipoamide dehydrogenase [WP_026234716] } \\
\text { Hypothetical protein [WP_018465799] }\end{array}$ & Reductase \\
\hline
\end{tabular}

neither of which contained any known component apart from the $\alpha$-subunit.

\section{Discussion}

Owing to their extensive presence among Proteobacteria and Actinobacteria, all extant ROs are postulated to have originated and evolved within these lineages (Chakraborty et al. 2014). Though the thermophilic RO homologs identified in this study were present among taxonomically close organisms, their distributions were very specific for certain phyla, often with very low abundance (Fig. 1). Thus, it is quite likely that the thermophiles also acquired $\mathrm{RO}_{\text {ox }}$ genes from Proteobacteria or Actinobacteria and further evolved separately. The role of Firmicutes in the evolution of ROs in thermophiles cannot be ruled out, as several RO homologs were found among mesophilic strains of Firmicutes (Fig. 1), especially within the order Bacillales.

Similarly, it would be difficult to claim that only the distantly located ET components identified in this study complement the putative $\alpha$ - and $\beta$-subunit genes. It is highly likely that unknown gene(s) vicinal to those 
encoding the terminal oxygenase(s) are responsible for the proper functioning of the ROs. Similar observations have previously been made in Rhodococcus opacus TKN14, in which rubredoxin and another hypothetical protein were found to be crucial for the oxidation of $o$-xylene (Maruyama et al. 2005). In another study, the purified large subunit of a novel alkane monooxygenase (belonging to Class B ROs), identified from a cold-tolerant Pusillimonas sp. T7-7, showed NADH-dependent alkane monooxygenase activity ( $\mathrm{Li}$ et al. 2013). Transformation of aromatic hydrocarbons has also been attained by heterologous expression of the terminal oxygenase components using non-specific ET proteins complemented by the host strain (Mukerjee-Dhar et al. 2005). Although the present study indicates that the RO homologs present in these organisms are either cryptic in nature or are involved in some other physiological function, we cannot rule out the possibility of reconstituting a thermostable functional RO system with novel properties (in terms of substrate preference or mode of catalysis) by combining the terminal oxygenase genes along with all possible combinations of ET components. Integrity of the motif signatures and predicted enhanced thermostability (Fig. 3) further strengthens this hypothesis.

The existence of unexplored microbial diversity, together with the availability of whole genomes, represents a large pool for future industrial catalysts. Thermostable ROs may be attractive candidates for carrying out efficient biotransformation at elevated temperatures. Apart from enhancing our understanding of the distribution of ROs in nature, the present study may aid in designing new bioremediation strategies or industrial biosynthetic processes. Based on the information provided here, functional RO systems can be reconstituted from each organism by cloning both terminal oxygenase and ET genes into suitable vectors and performing biotransformation assays using the predicted substrates (Fig. 4). On the other hand, gene knockout studies can be performed (provided that appropriate genetic tools are available) to help elucidate the physiological role of RO homologs with unknown functions in thermophilic bacteria.

\section{Additional file}

Additional file 1. Additional tables.

\section{Abbreviations}

$\mathrm{RO}$ : Rieske non-heme iron oxygenase; $\mathrm{RO}_{\text {ox: }}$ : large (a) subunit of $\mathrm{RO}$ terminal oxygenase; ET: electron transport; NCBI: National Center for Biotechnology Information; $\mathrm{NAD}(\mathrm{P}) \mathrm{H}$ : reduced form of nicotinamide adenine dinucleotide phosphate.
Authors' contributions

All the authors conceived the research work. JC conducted the analyses. JC, CSM, KO and $\mathrm{HN}$ analyzed the data. JC drafted the manuscript. All authors read and approved the final manuscript.

\section{Acknowledgements}

JC extends his thanks to the Biotechnology Research Center, The University of Tokyo, for providing research facilities.

\section{Competing interests}

The authors declare that they have no competing interests.

\section{Availability of data and materials}

The datasets supporting the conclusions of this article are included within the article and its additional file.

\section{Ethics approval}

This article does not contain any studies with human participants or animals performed by any of the authors.

\section{Funding}

This study was funded by the Japan Society for the Promotion of Science (JSPS). JC was supported by postdoctoral fellowship for foreign researchers from the JSPS.

Received: 8 November 2016 Accepted: 23 December 2016

Published online: 04 January 2017

\section{References}

Allen CR, Boyd DR, Dalton H, Sharma ND, Brannigan I, Kerley NA, Sheldrake NG, Taylor SC (1995) Enantioselective bacterial biotransformation routes to cis-diol metabolites of monosubstituted benzenes, naphthalene and benzocycloalkenes of either absolute configuration. Chem Commun 2:117-118. doi:10.1039/C39950000117

Altschul SF, Gish W, Miller W, Myers EW, Lipman DJ (1990) Basic local alignment search tool. J Mol Biol 215:403-410. doi:10.1016/S0022-2836(05)80360-2

Berim A, Park JJ, Gang DR (2014) Unexpected roles for ancient proteins: flavone 8-hydroxylase in sweet basil trichomes is a Rieske-type, PAO-family oxygenase. Plant J 80:385-395. doi:10.1111/tpj.12642

Boyd DR, Sharma ND, O'Dowd CR, Carroll JG, Loke PL, Allen CCR (2005) cisDihydrodiol, arene oxide and phenol metabolites of dictamnine: key intermediates in the biodegradation and biosynthesis of furoquinoline alkaloids. Chem Commun 31:3989-3991. doi:10.1039/b506944k

Bui VP, Hudlicky T, Hansen TV, Stenstrom Y (2002) Direct biooxidation of arenes to corresponding catechols with E. coli JM109(pDTG602). Application to synthesis of combretastatins A-1 and B-1. Tetrahedron Lett 43:2839-2841. doi:10.1016/S0040-4039(02)00389-1

Bult CJ, White O, Olsen GJ, Zhou L, Fleischmann RD, Sutton GG, Blake JA, FitzGerald LM, Clayton RA, Gocayne JD, Kerlavage AR, Dougherty BA, Tomb JF, Adams MD, Reich Cl, Overbeek R, Kirkness EF, Weinstock KG, Merrick JM, Glodek A, Scott JL, Geoghagen NS, Venter JC (1996) Complete genome sequence of the methanogenic archaeon, Methanococcus jannaschii. Science 273:1058-1073. doi:10.1126/science.273.5278.1058

Caliebe A, Grimm R, Kaiser G, Lübeck J, Soll J, Heins L (1997) The chloroplastic protein import machinery contains a Rieske-type iron-sulfur cluster and a mononuclear iron-binding protein. EMBO J 16:7342-7350. doi:10.1093/ emboj/16.24.7342

Chakraborty J, Ghosal D, Dutta A, Dutta TK (2012) An insight into the origin and functional evolution of bacterial aromatic ring-hydroxylating oxygenases. J Biomol Struct Dyn 30:419-436. doi:10.1080/07391102.201 2.682208

Chakraborty J, Jana T, Saha S, Dutta TK (2014) Ring-hydroxylating oxygenase database: a database of bacterial aromatic ring-hydroxylating oxygenases in the management of bioremediation and biocatalysis of aromatic compounds. Environ Microbiol Rep 6:519-523. doi:10.1111/1758-2229.12182

de Castro E, Sigrist CJ, Gattiker A, Bulliard V, Langendijk-Genevaux PS, Gasteiger E, Bairoch A, Hulo N (2006) ScanProsite: detection of PROSITE signature matches and ProRule-associated functional and structural residues in proteins. Nucleic Acids Res 34:W362-W365. doi:10.1093/nar/gkl124 
El-Sayed AK, Hothersall J, Cooper SM, Stephens E, Simpson TJ, Thomas CM (2003) Characterization of the mupirocin biosynthesis gene cluster from Pseudomonas fluorescens NCIMB 10586. Chem Biol 10:419-430. doi:10.1016/S1074-5521(03)00091-7

Ensley BD, Ratzkin BJ, Osslund TD, Simon MJ, Wackett LP, Gibson DT (1983) Expression of naphthalene oxidation genes in Escherichia coli results in the biosynthesis of indigo. Science 222:167-169. doi:10.1126/ science.6353574

Feitkenhauer H, Märk H (2003) Biodegradation of aliphatic and aromatic hydrocarbons at high temperatures. Water Sci Technol 47:123-130

Ferraro DJ, Gakhar L, Ramaswamy S (2005) Rieske business: structure-function of Rieske non-heme oxygenases. Biochem Biophys Res Commun 338:175-190. doi:10.1016/j.bbrc.2005.08.222

Gasteiger E, Hoogland C, Gattiker A, Duvaud S, Wilkins MR, Appel RD, Bairoch A (2005) Protein identification and analysis tools on the ExPASy server. In: Walker JM (ed) The proteomics protocols handbook. Humana Press, New York City, pp 571-607. doi: 10.1385/1-59259-890-0:571

Gibson DT, Parales RE (2000) Aromatic hydrocarbon dioxygenases in environmental biotechnology. Curr Opin Biotechnol 11:236-243. doi:10.1016/ S0958-1669(00)00090-2

Gibson DT, Subramanian V (1984). Microbial degradation of aromatic hydrocarbons. In: Gibson DT (ed) Microbial degradation of organic compounds. Dekker, Inc., New York, pp 181-252. doi: 10.1002/actp.1986.010371127

Gibson DT, Koch JR, Kallio RE (1968) Oxidative degradation of aromatic hydrocarbons by microorganisms. I. Enzymatic formation of catechol from benzene. Biochemistry 7:2653-2662. doi:10.1021/bi00847a031

Habe H, Omori T (2003) Genetics of polycyclic aromatic hydrocarbon metabolism in diverse aerobic bacteria. Biosci Biotechnol Biochem 67:225-243. doi:10.1271/bbb.67.225

Hedlund BP, Dodsworth JA, Murugapiran SK, Rinke C, Woyke T (2014) Impact of single-cell genomics and metagenomics on the emerging view of extremophile "microbial dark matter". Extremophiles 18:865-875. doi:10.1007/ s00792-014-0664-7

Hudlicky T, Gonzalez D, Gibson DT (1999) Enzymatic dihydroxylation of aromatics in enantioselective synthesis: expanding asymmetric methodology. Aldrichimica Acta 32:35-62

Ikai A (1980) Thermostability and aliphatic index of globular proteins. J Biochem 88:1895-1898

Iwai S, Chai B, Sul WJ, Cole JR, Hashsham SA, Tiedje JM (2010) Gene-targetedmetagenomics reveals extensive diversity of aromatic dioxygenase genes in the environment. ISME J 4:279-285. doi:10.1038/ismej.2009.104

Iwai S, Johnson TA, Chai B, Hashsham SA, Tiedje JM (2011) Comparison of the specificities and efficacies of primers for aromatic dioxygenase gene analysis of environmental samples. Appl Environ Microbiol 77:3551-3557. doi:10.1128/AEM.00331-11

Jiang H, Parales RE, Lynch NA, Gibson DT (1996) Site-directed mutagenesis of conserved amino acids in the alpha subunit of toluene dioxygenase: potential mononuclear non-heme iron coordination sites. J Bacteriol 178:3133-3139

Li P, Wang L, Feng L (2013) Characterization of a novel Rieske-type alkane monooxygenase system in Pusillimonas sp. strain T7-7. J Bacteriol 195:892-1901. doi:10.1128/JB.02107-12

Mallick S, Chakraborty J, Dutta TK (2011) Role of oxygenases in guiding diverse metabolic pathways in the bacterial degradation of low-molecular weight polycyclic aromatic hydrocarbons: a review. Crit Rev Microbiol 37:64-90. doi:10.3109/1040841X.2010.512268

Marchler-Bauer A, Panchenko AR, Shoemaker BA, Thiessen PA, Geer LY, Bryant SH (2002) CDD: a database of conserved domain alignments with links to domain three-dimensional structure. Nucleic Acids Res 30:281-283. doi:10.1093/nar/gkg087

Margesin R, Schinner F (2001) Biodegradation and bioremediation of hydrocarbons in extreme environments. Appl Microbiol Biotechnol 56:650-663. doi:10.1007/s002530100701

Maruyama T, Ishikura M, Taki H, Shindo K, Kasai H, Haga M, Inomata Y, Misawa N (2005) Isolation and characterization of o-xylene oxygenase genes from Rhodococcus opacus TKN14. Appl Environ Microbiol 71:7705-7715. doi:10.1128/AEM.71.12.7705-7715.2005

Mason JR, Cammack R (1992) The electron-transport proteins of hydroxylating bacterial dioxygenases. Annu Rev Microbiol 46:277-305. doi:10.1146/ annurev.mi.46.100192.001425
Meng YL, Wang YM, Zhang B, Nii N (2001) Isolation of a choline monooxygenase CDNA clone from Amaranthus tricolor and its expressions under stress conditions. Cell Res 11:187-193. doi:10.1038/sj.cr.7290085

Mukerjee-Dhar G, Shimura M, Miyazawa D, Kimbara K, Hatta T (2005) bph genes of the thermophilic PCB degrader, Bacillus sp. JF8: characterization of the divergent ring-hydroxylating dioxygenase and hydrolase genes upstream of the Mn-dependent BphC. Microbiology 151:4139-4151. doi:10.1099/mic.0.28437-0

Newman LM, Garcia H, Hudlicky T, Selifonov SA (2004) Directed evolution of the dioxygenase complex for the synthesis of furanone flavor compounds. Tetrahedron 60:729-734. doi:10.1016/j.tet.2003.10.105

Parales RE (2003) The role of active-site residues in naphthalene dioxygenase. J Ind Microbiol Biotechnol 30:271-278. doi:10.1007/s10295-003-0043-3

Perfumo A, Banat IM, Marchant R, Vezzulli L (2007) Thermally enhanced approaches for bioremediation of hydrocarbon-contaminated soils. Chemosphere 66:179-184. doi:10.1016/.j.chemosphere.2006.05.006

Reinbothe S, Quigley F, Gray J, Schemenewitz A, Reinbothe C (2004) Identification of plastid envelope proteins required for import of protochlorophyllide oxidoreductase A into the chloroplast of barley. Proc Natl Acad Sci USA 101:2197-2202. doi:10.1073/pnas.0307284101

Rottiers V, Motola DL, Gerisch B, Cummins CL, Nishiwaki K, Mangelsdorf DJ, Antebi A (2006) Hormonal control of C. elegans dauer formation and life span by a Rieske-like oxygenase. Dev Cell 10:473-482. doi:10.1016/j. devcel.2006.02.008

Saitou N, Nei M (1987) The neighbor-joining method: a new method for reconstructing phylogenetic trees. Mol Biol Evol 4:406-425

Schäfer F, Schuster J, Würz B, Härtig C, Harms H, Müller RH, Rohwerder T (2012) Synthesis of short-chain diols and unsaturated alcohols from secondary alcohol substrates by the Rieske nonheme mononuclear iron oxygenase MdpJ. Appl Environ Microbiol 78:6280-6284. doi:10.1128/AEM.01434-12

Shintani M, Ohtsubo Y, Fukuda K, Hosoyama A, Ohji S, Yamazoe A, Fujita N, Nagata Y, Tsuda M, Hatta T, Kimbara K (2014) Complete genome sequence of the thermophilic polychlorinated biphenyl degrader Geobacillus sp. strain JF8 (NBRC 109937). Genome Announc. doi:10.1128/ genomeA.01213-13

Sydor PK, Barry SM, Odulate OM, Barona-Gomez F, Haynes SW, Corre C, Song L, Challis GL (2011) Regio- and stereodivergent antibiotic oxidative carbocyclizations catalysed by Rieske oxygenase-like enzymes. Nat Chem 3:388-392. doi:10.1038/nchem.1024

Tamura K, Dudley J, Nei M, Kumar S (2007) MEGA4: molecular evolutionary genetics analysis (MEGA) software version 4.0. Mol Biol Evol 24:15961599. doi:10.1038/nchem.1024

Thompson JD, Gibson TJ, Plewniak F, Jeanmougin F, Higgins DG (1997) The CLUSTAL_X windows interface: flexible strategies for multiple sequence alignment aided by quality analysis tools. Nucleic Acids Res 25:48764882. doi:10.1093/nar/25.24.4876

Vieille C, Zeikus GJ (2001) Hyperthermophilic enzymes: sources, uses, and molecular mechanisms for thermostability. Microbiol Mol Biol Rev 65:1-43

Wackett LP, Kwart LD, Gibson DT (1988) Benzylic monooxygenation catalyzed by toluene dioxygenase from Pseudomonas putida. Biochemistry 27:1360-1367. doi:10.1021/bi00404a041

Wargo MJ, Szwergold BS, Hogan DA (2008) Identification of two gene clusters and a transcriptional regulator required for Pseudomonas aeruginosa glycine betaine catabolism. J Bacteriol 190:2690-2699. doi:10.1128/ JB.01393-07

Yoshiyama T, Namiki T, Mita K, Kataoka H, Niwa R (2006) Neverland is an evolutionally conserved Rieske-domain protein that is essential for ecdysone synthesis and insect growth. Development 133:2565-2574. doi:10.1242/ dev.02428

Yoshiyama-Yanagawa T, Enya S, Shimada-Niwa Y, Yaguchi S, Haramoto Y, Matsuya T, Shiomi K, Sasakura Y, Takahashi S, Asashima M, Kataoka H, Niwa R (2011) The conserved Rieske oxygenase DAF-36/Neverland is a novel cholesterol-metabolizing enzyme. J Biol Chem 286:25756-25762. doi:10.1074/jbc.M111.244384

Zezula J, Hudlicky T (2005) Recent progress in the synthesis of morphine alkaloids. Synlett 3:388-405. doi:10.1055/s-2005-862383 